\title{
Acaciaside-B-enriched fraction of Acacia auriculiformis is a prospective spermicide with no mutagenic property
}

\author{
Durba Pal ${ }^{1}$, Pratip Chakraborty ${ }^{3}, \mathrm{H} \mathrm{N} \mathrm{Ray}{ }^{1}, \mathrm{~B} \mathrm{C} \mathrm{Pal}^{2}$, Debashis Mitra ${ }^{4}$ and Syed N Kabir ${ }^{1}$ \\ ${ }^{1}$ Reproductive Biology Research and ${ }^{2}$ Department of Medicinal Chemistry, Indian Institute of Chemical Biology \\ (Council of Scientific and Industrial Research), Jadavpur, Kolkata 700032, West Bengal, India, ${ }^{3}$ Department of \\ Infertility, Institute of Reproductive Medicine, Salt Lake City, Kolkata 700091, West Bengal, India and ${ }^{4}$ Department \\ of Infection and Immunity, National Centre for Cell Science, Ganeshkhind, Pune 411007, India
}

Correspondence should be addressed to S N Kabir; Email: snkabir@iicb.res.in

\begin{abstract}
As a part of our continued venture to develop a safe and effective spermicide, we have identified a triterpene glycoside (Acaciaside-B (Ac-B))-enriched fraction (Ac-B-en) isolated from the seeds of Acacia auriculiformis and evaluated its spermicidal potential in vitro. Sperm motility was completely inhibited within $20 \mathrm{~s}$ at a minimum effective concentration (MEC) of $120 \mu \mathrm{g} / \mathrm{ml}$. Tests for sperm viability by dual fluoroprobe staining showed the effect to be spermicidal with an $\mathrm{EC}_{50}$ of $35.20 \mu \mathrm{g} / \mathrm{ml}$. A series of investigations including tests for hypo-osmotic swelling, membrane lipid peroxidation, and electron microscopy document that the spermicidal effect of the fraction involves loss of sperm plasma membrane integrity and dissolution of the acrosomal vesicle - the two most important structural components that play diverse roles in physiological functions of sperm including fertilization. The fraction at $10 \times \mathrm{MEC}$ exerted no detrimental effects on in vitro growth of Lactobacillus acidophilus, which is considered the major constituent of vaginal microflora that maintains vaginal health. Ames tests performed with different strains of Salmonella typhimurium including TA 97a, 98, 100, and 102, which detect mutagens causing bp substitution or frameshifting at G-C or A-T bp, demonstrate no mutagenic potential of the fraction. Significant spermicidal potential with no possible mutagenic effect and adverse impacts on lactobacilli growth attests to the credential of Ac-B-en as a prospective future spermicide for the development of a safe and effective vaginal contraceptive formulation.

Reproduction (2009) 138 453-462
\end{abstract}

\section{Introduction}

The population explosion has been a major global concern. Globally, over 200 million pregnancies occur every year and, of these, $\sim 50 \%$ are unwanted. A number of contraceptive options are available on the market, but successful prevention of unplanned pregnancies relies not only on access to available products, but also on the product's acceptability and couple's willingness and ability to use them effectively. Thus, in making contraceptive choices, couples balance their sexual lives, their reproductive goals, and each partner's health and safety. The search for a choice that satisfies all three objectives presents significant challenges for women and men throughout the world. Furthermore, what constitutes an ideal or suitable contraceptive method differs not only among individuals, but also as individuals enter different life phases. Therefore, it is important for women and men to have access to a wide variety of contraceptive options, which will allow them to select the suitable ones. Another major problem that has further complicated the situation is the increasing incidence of sexually transmitted infections (STIs) that mainly occurs through heterosexual contact. Women face the greatest risk of acquiring STIs because of substantial mucosal exposure to seminal fluids and the high prevalence of nonconsensual and unprotected sex (Smits et al. 1999, Clara et al. 2004). No cure exists for many STIs of viral etiology; particularly infections caused by human immunodeficiency virus (HIV) and herpes simplex virus. Consequently, prevention is the strategy of choice for controlling the spread of infection. This scenario has given rise to growing interest in combining contraception with STI prevention. The barrier methods such as condoms may prevent pregnancy and block HIV transmission when used appropriately; however, personal preferences and cultural practices have limited the use of condoms. There is a need for female-controlled methods to prevent unplanned pregnancy as well as infection. Development of vaginal microbicides with discerning spermicidal property, especially for formulations that may be available over the counter, constitutes one of the cornerstones of the prevention science agenda for curbing the rising HIV epidemic and unplanned pregnancy. The proposed chemical barriers can be used alone or in combination with a mechanical barrier to 
provide increased protection against pregnancy or STIs, or back-up in case of mechanical barrier failure. An ideal product would be female controlled, with minimal systemic exposure and adverse effects, and would have the ability to coat the vagina, cover the cervix and be retained for an extended period of time. The development of such products is of urgent need and has been the focus of a great deal of research activity for the last decade. Nonoxynol-9 (N-9), which was originally thought to have spermicidal as well as virucidal potential, was so far the most widely used effective molecule for spermicidal formulations. But N-9 has been subsequently shown to cause lesion of vaginal epithelium and damages the vaginal microflora to render the user vulnerable to STIs (Richardson et al. 1998). So the development of a suitable alternative is an urgent global need. Many novel compounds that have antifertility and possible antimicrobial effects are currently being developed and tested (Chaudhary et al. 2007). However, substantial challenges remain before a new contraceptive microbicide product will be available for distribution.

The contemporary trends in drug discovery for reproductive health care emphasize investigation of the terrestrial and marine environments. India, the centre of mega biodiversity with her varied climatic, altitudinal, and soil conditions, possesses about 3500 medicinal plants (Pal 1996). Recent efforts are being paid to explore these plants as a prospective source of spermicides.

Medicinal plants have long been known as a source of diverse kinds of saponins and terpenoids that represent the effective component of majority of the currently available spermicidal preparations (Farnsworth \& Waller 1982). Saponins, isolated from Indian medicinal plants, have been reported to have potential spermicidal properties (Setty et al. 1976). Acacia auriculiformis is a common Indian medicinal plant, the seeds of which are a rich source of triterpinoid saponins including Acaciaside-A (Ac-A) and Acaciaside-B (Ac-B). These acaciasides have been known for anti-helminthic activity, and can induce membrane damage by generating superoxide anions and initiating lipid peroxidation (Sinha Babu et al. 1997). Earlier investigations from this laboratory have demonstrated that an isolate of the extracts of $A$. auriculiformis seeds comprising of a mixture of Ac-A and Ac-B possesses spermicidal activity (Pakrashi et al. 1991). Subsequent studies have shown that Ac-A and Ac-B individually possess spermicidal properties; but Ac-A is a mutagen, while Ac-B is spermicidal at significantly lower concentrations, and also demonstrates anti-HIV potential in vitro but having no mutagenicity (D Pal, unpublished observations). However, from the practical point of view, the major limitation is its poor yield through extraction, and synthetic preparation of Ac-B is also not possible. During the extraction and isolation procedure, a crude fraction was obtained with high yield that is mostly comprised of Ac-B ( $>38 \%$ by weight) with no trace of Ac-A, henceforth referred to as
Ac-B-enriched (Ac-B-en) fraction. We took an interest in investigating the spermicidal potential and mutagenicity of Ac-B-en fraction and explored whether it can substitute pure Ac-B without compromising the efficacy.

\section{Results}

\section{Spermicidal potential}

The effect of test compounds on sperm motility has been presented in Fig. 1. With an increase in the concentration of Ac-B, Ac-B-en, and N-9 (not shown in the figure), there were dose-dependent increases in the immobilization of sperm. The minimum effective concentration (MEC) of Ac-B and Ac-B-en that induced $100 \%$ immobilization of sperm in $20 \mathrm{~s}$ was found to be 60 and $120 \mu \mathrm{g} / \mathrm{ml}$ respectively, as against $550 \mu \mathrm{g} / \mathrm{ml}$ for $\mathrm{N}-9$. In neither of the treated groups, any revival of motility was recorded following washing and incubation in Baker's buffer.

\section{Sperm viability assay}

Proportionate distribution of green- (live) and red- (dead) stained spermatozoa was recorded using dual emission filter for SYBR-14 and propidium iodide (PI). In the control set (without Ac-B-en treatment), 95\% were viable (green stained). With an increase in the concentration of Ac-B-en, there was a proportionate increase in the population of red-stained dead spermatozoa (Fig. 2). The presence of no viable spermatozoa was recorded following exposure to Ac-B-en at the concentration of $120 \mu \mathrm{g} / \mathrm{ml}$. A linearized dose-response curve was plotted (Fig. 3). The $\mathrm{EC}_{50}$ of Ac-B-en was calculated to be $35.20 \mu \mathrm{g} / \mathrm{ml}$.

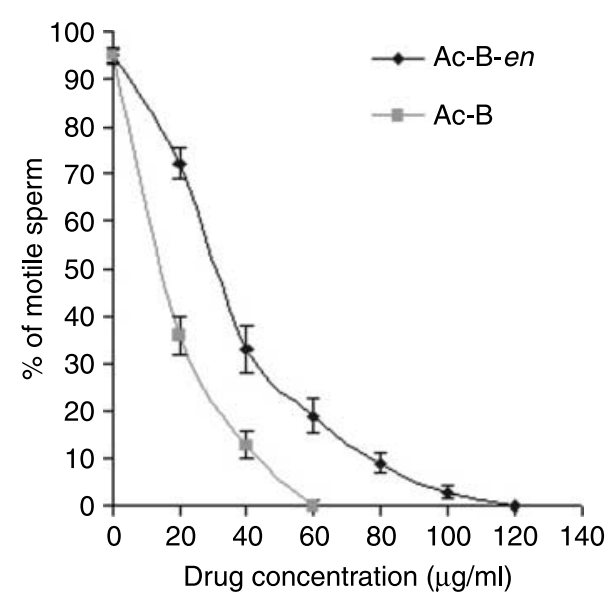

Figure 1 Dose-dependent sperm immobilizing activity of Ac-B and Ac-B-en. The percentage of motile human spermatozoa was determined after $20 \mathrm{~s}$ following exposure to the test compounds at different concentrations. All data were adjusted to a normal control motility of $95 \%$. Each point of AC-B represents the mean \pm s.D. values of five independent experiments; while there were 21 determinations against each concentration of Ac-B-en. 

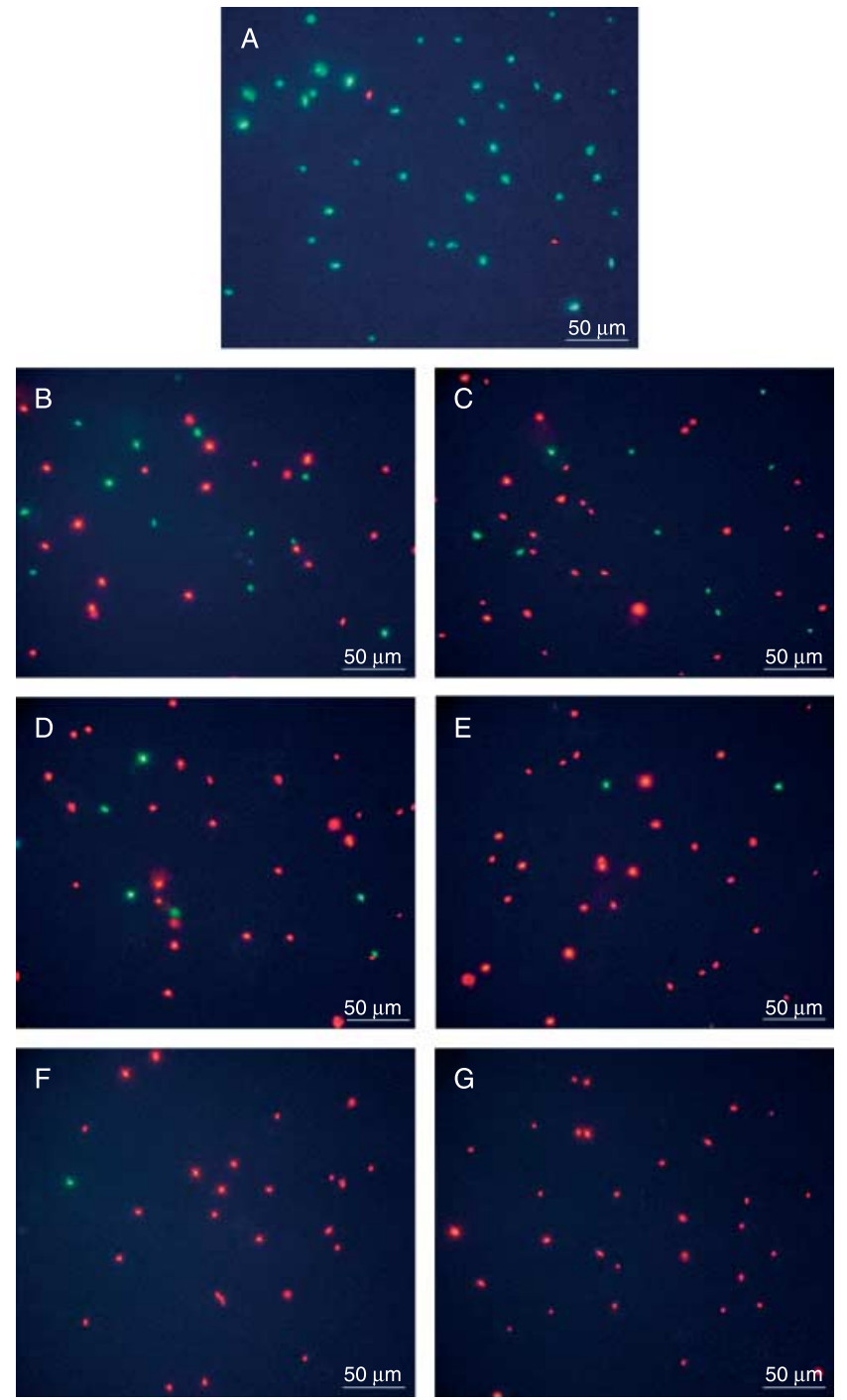

Figure 2 Overlaid fluorescence images of control and Ac-B-en-treated human spermatozoa, dual stained with SYBR-14 and propidium iodide to distinguish green-fluoresced live from red-fluoresced dead spermatozoa. The results demonstrate a proportionate decrease of live sperm in concert with increase in Ac-B-en concentrations ((A) $0 \mu \mathrm{g} / \mathrm{ml}$, (B) $20 \mu \mathrm{g} / \mathrm{ml}$, (C) $40 \mu \mathrm{g} / \mathrm{ml}$, (D) $60 \mu \mathrm{g} / \mathrm{ml}$, (E) $80 \mu \mathrm{g} / \mathrm{ml}$, (F) $100 \mu \mathrm{g} / \mathrm{ml}$, and $(\mathrm{G}) 120 \mu \mathrm{g} / \mathrm{ml}$ ). Each image is one representative of five replicates of the concerned subsets; bar $=50 \mu \mathrm{m}$.

\section{Effect on sperm membrane integrity}

Over $92 \%$ of the control spermatozoa responded to hypo-osmotic solution by swelling and curling of the tail, while $100 \%$ of the sperm treated with Ac-B-en at MEC exhibited no response to hypo-osmotic exposure (Fig. 4).

\section{Scanning electron microscopy}

Electron microscopic photographs of human sperm are presented in Fig. 5. As compared with intact plasma membrane and acrosomal vesicles of $92 \%$ untreated sperm, all (100\%) Ac-B-en-treated sperm exhibited disintegrated plasma membrane with damaged acrosomal cap of various degrees ranging from perforations and vesiculation to complete disintegration.

\section{Transmission electron microscopy}

Ultrastructural microphotograph showed considerable membrane damage in $100 \%$ of the Ac-B-en-exposed sperm. As compared with the intact plasma membrane surrounding the head of $84 \%$ of the control spermatozoa (Fig. 6A), all treated sperm exhibited dissolution of the acrosomal cap, expansion and separation of the plasma membrane from the nucleus (Fig. 6B).

\section{Effect on sperm lipid peroxidation}

The spectrophotometric readings demonstrated a dosedependent increase in the concentration of malondialdehyde (MDA; nmol/ $10^{8}$ sperm) in concert with an increase in Ac-B-en concentration (Fig. 7). As analyzed by ANOVA, the MDA production by sperm treated with MEC of Ac-B-en $\left(24.99 \pm 0.242 \mathrm{nmol} / 10^{8}\right.$ sperm) was significantly higher $(P<0.01)$ than that of control sperm $\left(20.66 \pm 0.266 \mathrm{nmol} / 10^{8}\right.$ sperm $)$.

\section{In vitro effect on Lactobacillus acidophilus}

Ac-B-en at $1 \times$ MEC $(120 \mu \mathrm{g} / \mathrm{ml})$ and $10 \times \mathrm{MEC}$ $(1.2 \mathrm{mg} / \mathrm{ml})$ did not significantly affect the growth of Lactobacillus colonies during the $36 \mathrm{~h}$ culture period. On the contrary, N-9 at $1 \times$ MEC $(550 \mu \mathrm{g} / \mathrm{ml})$ significantly inhibited the growth of Lactobacillus continuing throughout the $36 \mathrm{~h}$ span of the culture $(P<0.001$; Fig. 8).

\section{Test for mutagenicity}

Table 1 represents the number of revertant colonies in different treatment groups. Positive control plates, as expected, exhibited higher number of revertants over the spontaneous revertant rate for all tested strains.

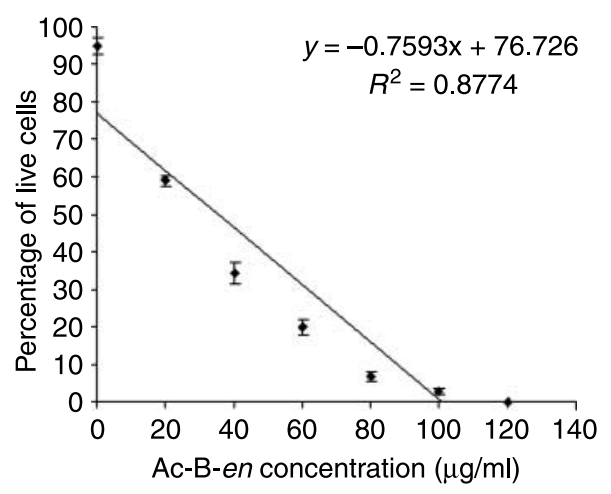

Figure 3 Proportionate number of live sperm over total sperm cells (\%), as evaluated by SYBR-14-PI dual staining, is plotted against the respective concentration of $\mathrm{Ac}-\mathrm{B}$-en. $\mathrm{EC}_{50}$ (concentration that induces death of $50 \%$ sperm) of Ac-B-en is calculated to be $35.20 \mu \mathrm{g} / \mathrm{ml}(n=5)$. 


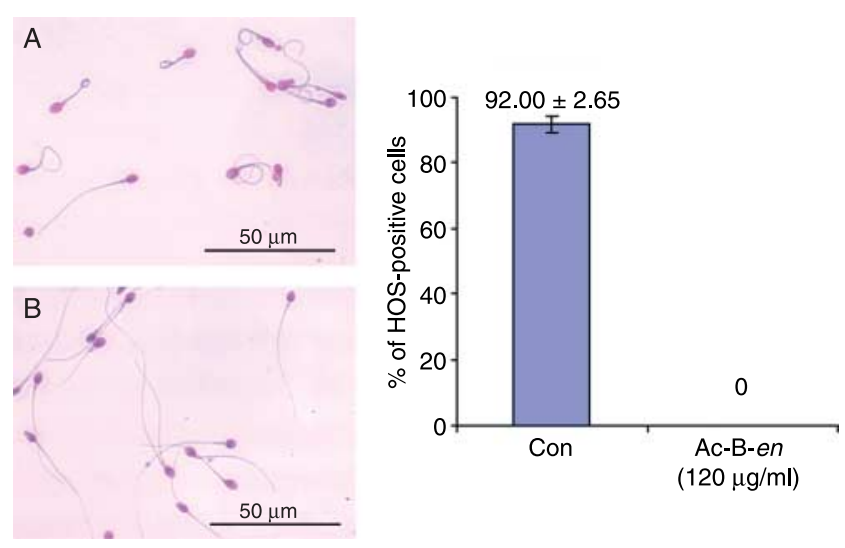

Figure 4 Response of control (A) and Ac-B-en-treated (B) human sperm population following exposure to hypo-osmotic solution and evaluated under a phase contrast microscope. Over $92 \%$ of control-untreated sperm exhibited HOS response typically characterized by tail coiling, whereas sperm exposed to Ac-B-en at MEC showed no response. Each bar represents the mean \pm s.D. of five observations. $P<0.0001$. Bar $=50 \mu \mathrm{m}$

In the Ac-B-en-treated groups, by contrast, the revertant colony sizes of neither of the bacterial strains differed significantly from those of the corresponding negative control, regardless of the presence or absence of S9 mix.

\section{Discussion}

With an overall objective to search for a spermicidal agent that may effectively serve as the active constituent of vaginal contraceptives, the present investigation evaluated Ac-B-en for its sperm-immobilizing efficacy in a series of in vitro experiments. The results document that Ac-B-en, like Ac-B, exerted a dose-dependent irreversible sperm-immobilizing effect. The MEC of Ac-B-en was found to be $120 \mu \mathrm{g} / \mathrm{ml}$, which was twice that of pure Ac-B, and the effect was adjudged spermicidal because no revival of sperm motility was demonstrated after the Ac-B-en-exposed sperm were incubated in Baker's buffer.

The plasma membrane plays a vital role in the process of sperm migration and fertilization (de Lamirande et al. 1997). A number of spermicidal agents are known to execute their effects by structural and functional modulation of the plasma membrane. We therefore took an interest in examining whether the spermicidal effect of Ac-B-en was mediated by adverse modulation of sperm membrane. Two commonly employed techniques were adopted: hypo-osmotic swelling (HOS) test; and dual staining with SYBR-14 and PI.

HOS is a response that reflects the functional integrity of sperm membrane (Jayendran et al. 1984). Following exposure to hypo-osmotic environment, the intact sperm membrane permits free passage of fluids into the cell to reach osmotic equilibrium. As a result, the sperm volume increases and plasma membrane bulges. Since the plasma membrane around the sperm tail fiber is more loosely attached than that around other parts, the sperm tail is particularly susceptible to hypo-osmotic exposure and responds by coiling. This characteristic feature was exhibited in $>92 \%$ of the sperm in the control set, while the Ac-B-en-exposed sperm showed no such morphological distortion. This observation suggests that the functional integrity of the sperm membrane was lost following exposure to Ac-B-en. This suggestion was additionally supported by the differential reaction of the normal and Ac-B-en-exposed sperm to a living cell nucleic acid stain SYBR-14 and a membraneimpermeable dye PI. A morphologically intact membrane in live sperm offers selective permeability and, therefore, debars entry of fluorescent dye like Pl. But as the sperm die, they lose their ability to resist the influx of $\mathrm{PI}$, which upon entering the sperm replaces or quenches the SYBR14 staining and turns the sperm red. This forms the basis of the dual staining technique to differentiate between live and dead sperm (Garner \& Johnson 1995). We observed that following dual fluorescent staining, almost $\sim 5 \%$ of the total population of control spermatozoa showed PI staining, while the remainders appeared entirely green due to SYBR-14 staining only. By contrast, exposure to gradually increased concentration of Ac-B-en produced a gradual and proportional decrease in SYBR-14-stained green sperm population with parallel increase in Pl-stained red sperm. At $120 \mu \mathrm{g} / \mathrm{ml}$ concentration of Ac-B-en, all sperm were positively stained with PI. Taken

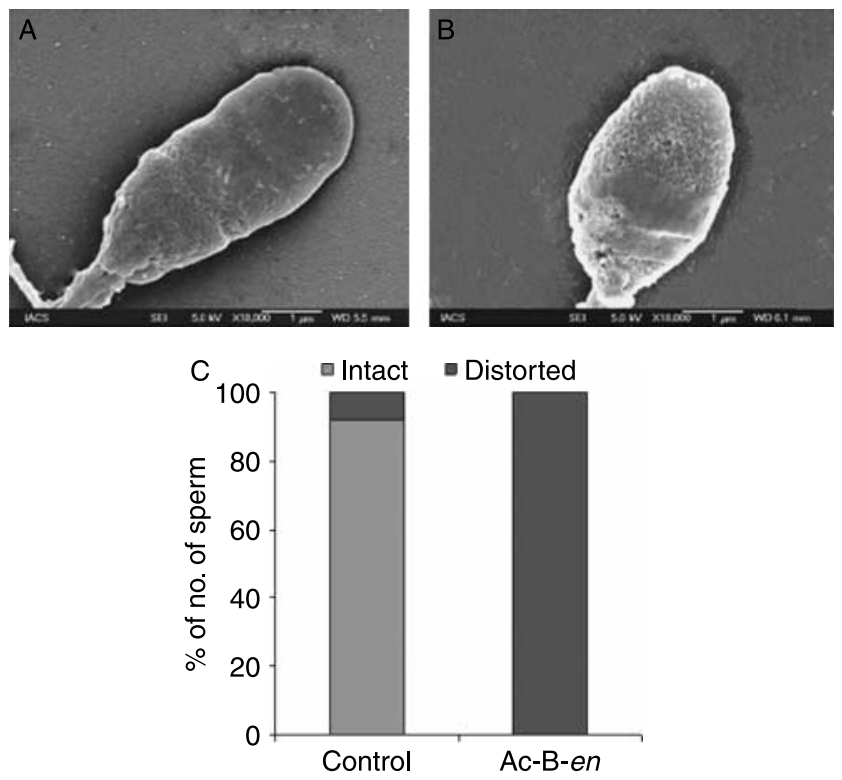

Figure 5 High resolution scanning electron micrographs $(\times 18000)$ of human sperm treated without and with Ac-B-en at MEC. (A) Control sperm shows intact acrosomal cap and plasma membrane around the head and neck regions, while (B) Ac-B-en-treated sperm demonstrates dissolution of the acrosomal cap; (C) histogram shows increased percentage $(P<0.0001)$ of sperm with distorted acrosome in the Ac-B-en-treated groups. The values are based on examining 50 sperm in each group. Bar $=1 \mu \mathrm{m}$. 

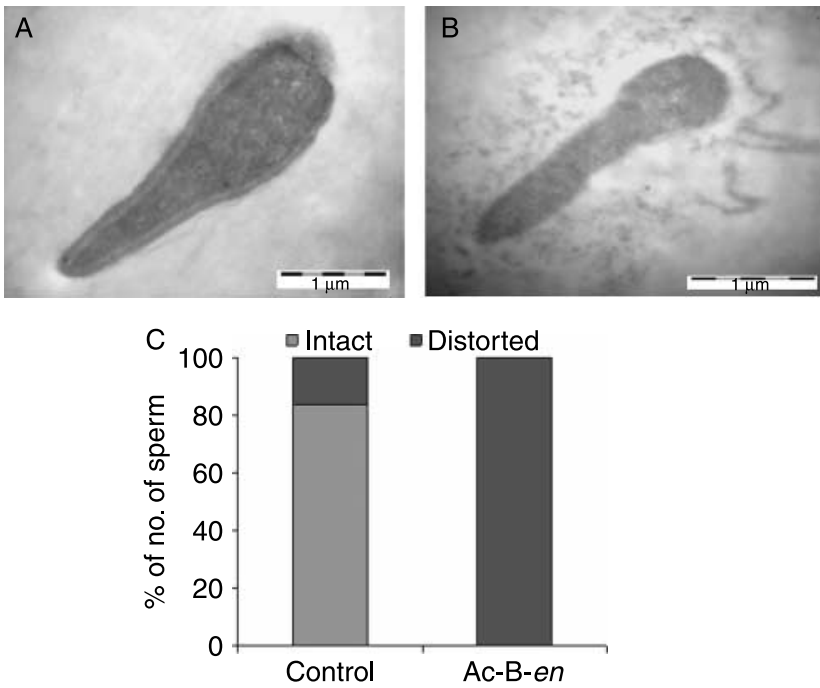

Figure 6 Transmission electron micrographs $(\times 100000)$ of human sperm samples incubated in the absence or presence of Ac-B-en (at MEC). (A) Control spermatozoa show proper acrosomal cap with intact plasma membrane, while (B) Ac-B-en-treated spermatozoa exhibit dissolution of the acrosomal cap; $(C)$ histogram shows increased percentage $(P<0.0001)$ of distorted sperm in the Ac-B-en-treated group. The values are based on examining 50 sperm in each group.

together, these observations left no doubt that Ac-B-en led to the loss of functional and morphological integrity of the sperm membrane. Consistent with these results, the electron microscopy findings also demonstrated that the effect of Ac-B-en on sperm membrane involved the loss of plasma membrane architecture with dissolution of the outer acrosomal membrane.

Nonionic surfactants are amphipathic molecules consisting of a hydrophobic (alkylated phenol derivatives, fatty acids, long-chain linear alcohols, etc.) and a hydrophilic part (generally ethylene oxide chains of various length). Due to this favorable physicochemical property, nonionic surfactants interact not only with proteins but also with membrane phospholipids to modify their structure and permeability. Mammalian sperm cells present highly specific lipid composition comprising a high proportion of polyunsaturated fatty acids, plasmalogens, and sphingomyelins. This unusual structure of the sperm membrane renders it highly susceptible to damage by nonionic surfactants. It was suggested that the double bonds present in nonionic surfactants form allelic radicals that may react with the molecular oxygen present in the membrane and cause lipid peroxidation (Nandi et al. 2004). This mechanism perhaps explains the increased lipid peroxidation of the sperm membrane preparation and loss of membrane integrity under the exposure to Ac-B-en.

One major shortcoming of the presently marketed spermicides is their adverse effects on vaginal ecology. The vagina and cervix constitute a complex ecosystem that includes the vaginal and cervical epithelia, microbial flora, vaginal fluid, and cervical mucus. The normal vaginal flora of healthy women of childbearing age is dominated by lactobacilli. Lactobacillus produces a number of compounds including lactic acid, hydrogen peroxide, lactacin, and acidolin that maintain a low, acidic pH (3.5-5.0; Hawes et al. 1996), and thereby protect against the pathogens that cause STIs including HIV (Kempf et al. 1991, Klebanoff \& Coombs 1991). In order to predict any possible adverse effects of Ac-B-en on vaginal microflora, we investigated the impacts of the test fraction on the growth of L. acidophilus, a major species that represents the vaginal lactobacilli population. The present study demonstrates that as compared with control, Ac-B-en upto a tenfold concentration of MEC did not inhibit the growth of $L$. acidophilus colonies during the period of $36 \mathrm{~h}$ of culture. It may therefore be envisioned that Ac-B-en, unlike the existing spermicides, possibly would not disturb the vaginal ecology to invite opportunistic infections. However, the L. acidophilus complex is highly heterogeneous and varies between geographically separated locations (Vásquez et al. 2002). Ac-B-en may be effective against a particular species, but its action against other strains or subspecies of Lactobacillus may be investigated to give more power to the prediction of safety against commensal microorganisms of the human vagina covering different geographical locations.

Because of the intimate contact of a spermicidal agent with human gametes and the possibility that components of the spermicide may be absorbed systemically raise concerns about the possible mutagenic effect of the test substances (Lambert et al. 2004). We evaluated the possibility of mutation via the bacterial reverse mutation test in which histidine-requiring strains of Salmonella typhimurium were used to detect point mutations involving subsititution, addition, or deletion of one or few DNA bp. Each of the four strains of Salmonella

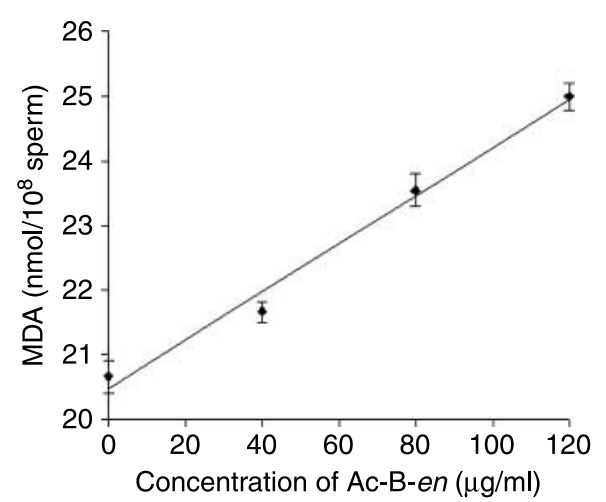

Figure 7 Generation of malondialdehyde (MDA) as a function of lipid peroxidation by human sperm treated with or without Ac-B-en at varying concentrations. Values on $Y$-axis represent mean \pm s.D. value of five determinations. The graph shows dose-dependent increase in the MDA generation following exposure of motile spermatozoa to Ac-B-en $(P<0.01)$. 


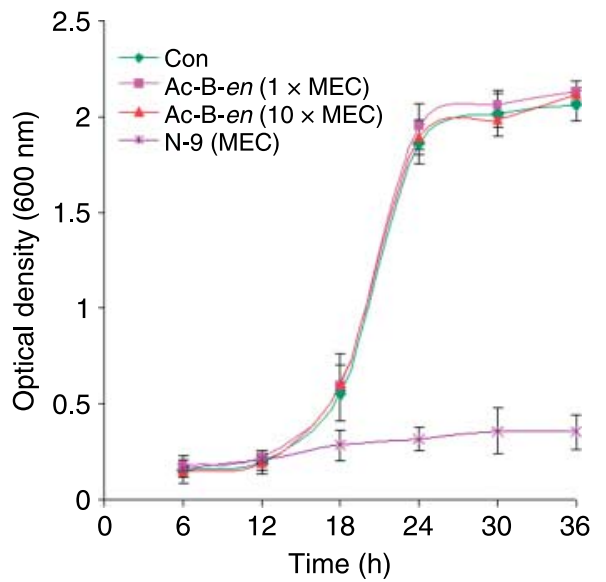

Figure 8 Optical density as the measure of turbidity denoting growth of bacteria colonies during $36 \mathrm{~h}$ of culture in the absence (control) and presence of different test compounds. There was a gradual increase in the growth of colonies that reached a plateau after $24 \mathrm{~h}$ of culture. Irrespective of the dose of Ac-B-en $(1 \times$ MEC and $10 \times M E C)$, the growth of bacterial colony was comparable with that of the control. N-9, however, exerted a constant inhibition over bacterial growth throughout the entire culture period. Each points represent the mean \pm s.D. value of five independent cultures.

contains a different type of mutation in the histidine operon. S. typhimurium TA100 detects mutagens that cause bp substitutions at G-C pairs; TA 97a and TA 98 detect frameshift mutagens that damage the correct reading frame of histidine synthesis at G-C bp (Halder et al. 2005). Since Ac-B-en increases lipid peroxidation and these strains may not detect the oxidizing mutagens, we also employed the strain TA102 (Windebank et al. 2006), which has A-T bp in the primary reversion site. The test was performed with or without metabolic activities by cofactor-supplemented post-mitochondrial fraction (S9). For all test strains, regardless of the presence or absence of $\mathrm{S} 9 \mathrm{mix}$, the number of revertant colonies in the Ac-B-en-treated groups did not differ significantly from that in the corresponding negative control. The reproducibility of the test results was confirmed. The positive controls used in the assays in the presence or absence of S9 mix showed clear positive responses by the respective test strains, as evidenced by the number of revertant colonies being greater than twofold of the respective negative control value. From the results described above, it may be concluded that Ac-B-en is expected to be safe in terms of mutagenicity.

We further emphasize that although Ac-B-en, like the most popularly used spermicide $\mathrm{N}-9$, is a nonionic surfactant; it differs from N-9 in various respects. Ac-B is a natural compound having molecular weight 1876, which is about threefold that of the synthetic molecule N-9 (molecular weight 617), which indicates a lesser chance of its absorption through vaginal epithelia. Also, the MEC is about fourfold lower than that of $\mathrm{N}-9$ $(550 \mu \mathrm{g} / \mathrm{ml})$. Moreover, N-9-containing preparations

Table 1 Mutagenicity test outcome (number of revertant colonies) in Salmonella typhimurium strains TA97a, TA98, TA100, and TA102 on exposure to Ac-B-en with and without S9 activation.

\begin{tabular}{|c|c|c|}
\hline \multirow[b]{2}{*}{ Salmonella strains } & \multicolumn{2}{|c|}{ Revertant colony size $(\text { mean } \pm \text { s.D. })^{a}$} \\
\hline & Without S-9 activation & With S-9 activation \\
\hline \multicolumn{3}{|l|}{ TA 97a } \\
\hline Control (DMSO) & $54.33 \pm 6.51^{*}$ & $58.5 \pm 5.97 *$ \\
\hline Positive control (4-NPD/B(a)P) & $295.70 \pm 8.96^{+}$ & $146.5 \pm 20.01^{\dagger}$ \\
\hline Ac-B-en $(0.12 \mathrm{mg} / \mathrm{ml})$ & $49.0 \pm 6.08^{*}$ & $54.5 \pm 6.35^{*}$ \\
\hline Ac-B-en $(1.20 \mathrm{mg} / \mathrm{ml})$ & $51.33 \pm 4.04 *$ & $56.0 \pm 3.92 *$ \\
\hline Ac-B-en (12 mg/ml) & $52.0 \pm 6.25^{*}$ & $58.0 \pm 4.32 *$ \\
\hline \multicolumn{3}{|l|}{ TA 98} \\
\hline Control (DMSO) & $21.75 \pm 1.71^{*}$ & $19.5 \pm 6.56^{*}$ \\
\hline Positive control (4-NPD/B(a)P) & $230.30 \pm 12.92^{+}$ & $95.5 \pm 9.57^{+}$ \\
\hline Ac-B-en $(0.12 \mathrm{mg} / \mathrm{ml})$ & $19.75 \pm 6.65^{*}$ & $19.1 \pm 5.88^{*}$ \\
\hline Ac-B-en $(1.20 \mathrm{mg} / \mathrm{ml})$ & $20.5 \pm 3.42 *$ & $18.6 \pm 3.42 *$ \\
\hline Ac-B-en $(12 \mathrm{mg} / \mathrm{ml})$ & $15.25 \pm 4.57 *$ & $18.0 \pm 2.94^{*}$ \\
\hline \multicolumn{3}{|l|}{ TA 100} \\
\hline Control (DMSO) & $66.0 \pm 12.53^{*}$ & $65.67 \pm 6.11^{*}$ \\
\hline Positive control $\left(\mathrm{NaN}_{3} / \mathrm{B}(\mathrm{a}) \mathrm{P}\right)$ & $387.7 \pm 12.58^{+}$ & $494.3 \pm 14.57^{+}$ \\
\hline Ac-B-en $(0.12 \mathrm{mg} / \mathrm{ml})$ & $68.33 \pm 9.02 *$ & $66.33 \pm 6.11^{*}$ \\
\hline Ac-B-en $(1.20 \mathrm{mg} / \mathrm{ml})$ & $68.0 \pm 2.00^{*}$ & $66.67 \pm 4.73^{*}$ \\
\hline Ac-B-en (12 mg/ml) & $67.33 \pm 3.51^{*}$ & $64.1 \pm 11.00^{*}$ \\
\hline \multicolumn{3}{|l|}{ TA 102} \\
\hline Control (DMSO) & $118.0 \pm 7.94 *$ & $121.1 \pm 6.31^{*}$ \\
\hline Positive control (Daunomycin/B(a)P) & $269.0 \pm 12.12^{+}$ & $453.6 \pm 15.20^{+}$ \\
\hline Ac-B-en $(0.12 \mathrm{mg} / \mathrm{ml})$ & $116.0 \pm 5.00^{*}$ & $115 \pm 2.38^{*}$ \\
\hline Ac-B-en $(1.20 \mathrm{mg} / \mathrm{ml})$ & $110.7 \pm 15.53^{*}$ & $119 \pm 8.70^{*}$ \\
\hline Ac-B-en $(12 \mathrm{mg} / \mathrm{ml})$ & $117.3 \pm 4.16^{*}$ & $117 \pm 5.24^{*}$ \\
\hline
\end{tabular}

${ }^{*}, \mathrm{t}$ Values with different superscripts against each strain under identical culture condition differ significantly $(P<0.001)$. 4-NPD, 4-nitrophenylene diamine; $\mathrm{NaN}_{3}$, sodium azide; $\mathrm{B}(\mathrm{a}) \mathrm{P}$, benzo( $\alpha$ ) pyrene.

${ }^{a}$ Represents mean \pm s.D. counts of five representative plates. 
disturb the vaginal microflora by inhibiting the growth of lactobacilli, leading to an increased risk of developing consequential infections in the genitourinary tract (Richardson et al. 1998); and several studies have documented that N-9-containing vaginal spermicide formulations are mutagenic both with and without liver activation (Lambert et al. 2004). Thus, Ac-B-en appears superior to N-9 with respect to effective concentration, mutagenicity, and vaginal health.

Kabir et al. (2008) have demonstrated that Ac-B inhibits transmission of HIV in vitro at concentrations lower than that of its cytotoxic doses. HIV is known to require intact lipid rafts (highly specialized subregions in cell membranes) for entry into cells and budding of fully infectious particles (Campbell et al. 2001). The lipiddispersing effect of Ac-B that may disrupt the lipid rafts is postulated to attribute to the anti-HIV property of Ac-B (Kabir et al. 2008). It is also important to note that Ac-B at doses 100 -folds of hemolytic index did not show any toxicity on the vaginal epithelium (HN Ray, unpublished observations). Since Ac-B represents the major constituent of Ac-B-en, it seems probable that Ac-B-en would be safe for vaginal epithelia; and its possible anti-HIV potential seems worthy to be explored.

Taking all observations into consideration, we conclude that significant spermicidal activity with apparently no possible mutagenic or adverse effects on vaginal ecology highlights the credentials of Ac-B-en as a prospective alternative to pure Ac-B for the development of spermicidal formulation. However, nonclinical pharmacology/toxicology issues and regulatory considerations that are pertinent to the development of topical spermicides need to be addressed.

\section{Materials and Methods}

\section{Isolation of Ac-B-enriched fraction}

The air-dried and powdered seeds $(1.5 \mathrm{~kg})$ of $A$. auriculiformis were extracted with methanol $(4 \mid \times 3)$ by percolation. Methanol extract was evaporated to dryness under reduced pressure. The residue $(73 \mathrm{~g})$ was suspended in water and extracted successively with ethyl acetate and $n$-butanol $(1 \mathrm{I} \times 3)$. The solutions were evaporated in vacuo to provide ethyl acetate-soluble (8 g), $n$-butanol-soluble (38 g), and watersoluble portions $(46 \mathrm{~g})$. A portion $(25 \mathrm{~g})$ of the $n$-butanol fraction was chromatographed on silica gel with petroleum ether, petroleum ether-chloroform (1:1), chloroform, and chloroform-methanol $(9: 1,4: 1,7: 3,3: 2,1: 1$ and 2:3) as successive eluents. The chloroform-methanol (7:3 and 3:2) eluates $(3.0 \mathrm{~g})$ were combined and subjected to rechromatography. Thus, a thin layer chromatographic homogeneous glycoside fraction $(2.5 \mathrm{~g})$ was obtained. This fraction was found to be a mixture of mainly two compounds by HPLC analysis. Preparative HPLC of this glycoside mixture with the solvent system methanol-water $(7: 3)$ at a flow rate of $10 \mathrm{ml} / \mathrm{min}$ afforded Ac-A (1.21 g) and Ac-B (0.85 g).
Enriched Ac-B was prepared from the water-soluble fraction. A solution of this fraction (46 g) in a small amount of water was poured into a large amount of $n$-butanol to form a precipitate. The precipitate was filtered and dried in a vacuum desiccator. This process was repeated three times to get Ac-B-en (18 g).

\section{Chemicals}

Unless otherwise stated, chemicals used for the preparation of reagents were of analytic grade and purchased from SigmaAldrich. Purified water (Milli-Q Biocel System, Millipore Corporation, Bedford, MA, USA) was used throughout the investigations. Disposable plastic wares were purchased from Tarsons Products Pvt Ltd (Kolkata, India). The media for culture of bacteria were purchased from Hi-Media Laboratories Pvt Ltd, Mumbai, India. Dual fluorescent live/dead staining kit was purchased from Invitrogen. The mutagenicity test was performed by using Oxoid nutrient broth No. 2 (Remel Inc., Lenexa, KS, USA). All common chemicals including sodium chloride $(\mathrm{NaCl})$, potassium chloride $(\mathrm{KCl})$, calcium chloride $\left(\mathrm{CaCl}_{2}\right)$, potassium dihydrogen phosphate $\left(\mathrm{KH}_{2} \mathrm{PO}_{4}\right)$, disodium hydrogen phosphate $\left(\mathrm{Na}_{2} \mathrm{HPO}_{4}\right)$, magnesium sulphate $\left(\mathrm{MgSO}_{4}\right)$, sodium bicarbonate $\left(\mathrm{NaHCO}_{3}\right)$, fructose, sodium citrate, thiobarbituric acid (TBA), and trichloroacetic acid (TCA) were purchased from Merck Specialities Pvt Ltd (Mumbai, India).

\section{Working media}

Working spermatozoa suspensions were made in Biggers, Whitten, and Whittingham's medium (BWW; $94 \mathrm{mM} \mathrm{NaCl}$, $4.7 \mathrm{mM} \mathrm{KCl}, 1.7 \mathrm{mM} \mathrm{CaCl}, 1.2 \mathrm{mM} \mathrm{KH_{2 }} \mathrm{PO}_{4}, 1.2 \mathrm{mM}$ $\mathrm{MgSO}_{4} \cdot 7 \mathrm{H}_{2} \mathrm{O}, 25 \mathrm{mM} \mathrm{NaHCO}, 0.5 \mathrm{mM}$ sodium pyruvate, $19 \mathrm{mM}$ sodium lactate, $5 \mathrm{mM}$ glucose, $0.4 \% \mathrm{BSA}$, and $0.1 \%$ antibiotic (penicillin/streptomycin) solution, $\mathrm{pH} 7.2$ ).

\section{Semen collection and processing}

Human semen samples, collected by masturbation after 3-4 days of sexual abstinence, were obtained from Institute of Reproductive Medicine, Kolkata, with due approval of the ethics committee. Donors gave informed, written consent. Samples having $>60 \times 10^{6} / \mathrm{ml}$ sperm count with $>85 \%$ motility and normal morphology were used for the study. The highly motile spermatozoa with forward motility were washed with BWW medium, separated from immotile or sluggishly motile cells by the 'swim-up' technique (WHO 1999), and finally resuspended in pre-equilibrated BWW medium to obtain working spermatozoa suspension having concentration of $25-30 \times 10^{6}$ cells $/ \mathrm{ml}$.

\section{Determination of spermicidal activity}

The spermicidal effect of Ac-B and Ac-B-en was evaluated by a modified version of Sander and Cramer test (Sander \& Cramer 1941) followed by test for reversal of motility. Solutions of pure $\mathrm{Ac}-\mathrm{B}$ and $\mathrm{Ac}-\mathrm{B}-\mathrm{en}$ having concentrations ranging between 10 and $200 \mu \mathrm{g} / \mathrm{ml}$ were prepared by serial dilutions in BWW 
medium. An aliquot of $20 \mu \mathrm{l}$ of sperm suspension was mixed with $100 \mu \mathrm{l}$ of BWW medium (control), or each concentration of Ac-B and Ac-B-en solution at a ratio 1:5 and gently vortexed for 1-2 s. A drop was immediately placed on a glass slide, covered with a cover slip and five different fields were quickly examined under a phase contrast microscope $(\times 100)$. The results were observed for $20 \mathrm{~s}$ and counted for motile sperm. The sperm that lost complete motility within $20 \mathrm{~s}$ following exposure to drugs were subsequently tested for motility revival.

The spermatozoa treated with Ac-B and Ac-B-en at 60 and $120 \mu \mathrm{g} / \mathrm{ml}$ respectively, which induced immobilization of $100 \%$ sperm, were washed twice in pre-equilibrated BWW medium, resuspended in fresh $250 \mu$ of Baker's buffer (glucose $3 \%, \mathrm{Na}_{2} \mathrm{HPO}_{4} \cdot 2 \quad \mathrm{H}_{2} \mathrm{O} 0.31 \%, \mathrm{NaCl} 0.2 \%$, and $\mathrm{KH}_{2} \mathrm{PO}_{4}$ $0.01 \%$ ), and incubated at $37^{\circ} \mathrm{C}$ for $60 \mathrm{~min}$. At the end of the incubation, a wet preparation of the treated spermatozoa from each set was made on a glass slide. The preparation was examined under a phase contrast microscope (at 100X) to observe any recovery of motility. Even if a single sperm in the ten fields that were examined showed any sign of jerking or viability, the dose was not recorded as 'effective dose'. The MEC of Ac-B-en that caused $100 \%$ immobilization within $20 \mathrm{~s}$ with no subsequent revival of motility was considered to be the MEC.

\section{Sperm viability assay}

Effect of Ac-B-en on viability of human spermatozoa was evaluated using a dual fluorescent live/dead staining kit consisting of SYBR-14 and PI (Flajshans et al. 2004). Briefly, a 50-fold dilution of the SYBR-14 stock solution was prepared in anhydrous DMSO. Sperm suspensions were treated with $100 \mu \mathrm{l}$ solution of Ac-B-en of varying concentrations $(0-120 \mu \mathrm{g} / \mathrm{ml})$ or diluted 1:5 with BWW medium (control), identically to that during determination of spermicidal activity by Sander-Cramer method. Immediately after $20 \mathrm{~s}, 2 \mathrm{ml}$ of BWW medium were added, and sperm were washed and centrifuged twice. The sperm pellet was resuspended in $1 \mathrm{ml}$ BWW medium to which $5 \mu \mathrm{l}$ of diluted SYBR-14 dye were added. After $5 \mathrm{~min}$ of incubation, $5 \mu \mathrm{l}$ of $\mathrm{PI}$ were added and finally incubated for $10 \mathrm{~min}$ at $37^{\circ} \mathrm{C}$. The fluorescent staining of sperm was monitored and photographed with a Zeiss Axiophot epifluorescent microscope (Carl Zeiss Inc., Thornwood, NY, USA) equipped with a FITC filter set (Zeiss \#487909). The proportionate number of live sperm over total number of cells $(\%)$ which was graphically plotted against each concentration of the test solution, was linealized and $\mathrm{EC}_{50}$ (the concentration that induced death of $50 \%$ spermatozoa) was assessed. Each point on $Y$-axis represents mean \pm s.D. (\%) of five independent observations with respective concentrations of Ac-B-en.

\section{HOS test}

The physiological integrity of the sperm plasma membrane was evaluated by HOS test (Jayendran et al. 1984) using the kit developed by National Institute of Health \& Family Welfare, New Delhi, India, for sperm function tests. Sperm were treated without (control) or with Ac-B-en at MEC for $20 \mathrm{~s}$ and exposed to $500 \mu \mathrm{l}$ of hypo-osmotic solution. This was mixed gently and incubated at room temperature for $5 \mathrm{~min}$ followed by the addition of $50 \mu \mathrm{l}$ of color stop solution. After thorough mixing, a tiny drop of the mixture was placed on a glass slide and covered with cover slip. The number of spermatozoa exhibiting characteristic swelling or tail coiling was counted in a total population of 1000 sperm per specimen under a phase contrast microscope $(\times 400)$. The percentage of HOS response was calculated by counting the number of sperm showing the characteristic morphologic changes divided by total number of sperm and multiplied by 100 . The data were collected from five independent sets of experiment and presented as mean \pm s.D.

\section{Electron microscopy}

\section{High resolution scanning electron microscopy}

Topographical imaging of membrane domains over the sperm head (Wilborn et al. 1983) was done by high resolution scanning electron microscopy (SEM). Control (untreated) human spermatozoa and Ac-B-en-exposed ones at MEC were fixed in $1 \%$ paraformaldehyde and $1 \%$ glutaraldehyde in $0.05 \mathrm{M}$ phosphate buffer (PB; $\mathrm{pH}$ 7.2) for $3 \mathrm{~h}$ followed by alternate spinning (5 min at $900 \mathrm{~g}$ ) and washing for three times. After the final washing, suspensions were placed on $0.1 \%$ poly-L-lysine-coated glass chips and allowed to adhere for $1 \mathrm{~h}$ at room temperature. Samples were post-fixed in $1 \%$ osmium tetraoxide in $0.5 \mathrm{M} \mathrm{PB}$ for $1 \mathrm{~h}$ at room temperature and subsequently dehydrated through an ascending series of ethanol, critical point dried and coated with Au-Pd (80:20) using a sputter coater (Emitech K-575X). All samples were examined using a Field Emission SEM (Philips FEI) at an accelerating voltage of $30 \mathrm{kV}$. Spermatozoa were observed initially under low magnification $(\times 2000-5000)$ and representative spermatozoa were photographed under intermediate magnification $(\times 18000-20000)$. For each group, 50 spermatozoa were scanned for the intactness of the acrosomal region. The difference between the treated and untreated groups with respect to intactness of the acrosomal vesicle was done by $\chi^{2}$-test.

\section{Transmission electron microscopy}

Transmission electron microscopy was performed according to the method as described by Souada et al. (2007). Briefly, control (untreated) and Ac-B-en-treated spermatozoa were fixed in $2.5 \%$ glutaraldehyde in $0.2 \mathrm{M} \mathrm{PB}(\mathrm{pH} 7.4)$ for $2 \mathrm{~h}$ at $4{ }^{\circ} \mathrm{C}$, and then post-fixed overnight in $1 \%$ osmium tetraoxide. The sample was dehydrated in alcohol, embedded in spurr resins, and polymerized for $48 \mathrm{~h}$. Ultra thin sections were cut and stained with uranyl acetate and lead citrate and observed under the transmission electron microscope (Philips TECHNEI) at low $(\times 25000)$ and high $(\times 100000)$ magnification. Fifty sperm from the untreated as well as treated groups were evaluated for the intactness of sperm head membranes and photograph of one representative sperm from each group has been presented. The difference between the treated and untreated groups with respect to intactness of the sperm head membranes was done by $\chi^{2}$-test. 


\section{Determination of lipid peroxidation}

The magnitude of lipid peroxidation of sperm was measured by determining MDA production using TBA as per the method of Buege \& Aust (1978) subsequently modified by Suleiman et al. (1996). Ac-B-en was serially diluted in BWW medium to make solutions of final concentrations ranging between 20 and $120 \mu \mathrm{g} / \mathrm{ml}$ and mixed with sperm suspension at 5:1 ratio. Briefly, $1 \mathrm{ml}$ suspension of spermatozoa $\left(25-30 \times 10^{6} \mathrm{cell} / \mathrm{ml}\right)$ treated with or without different concentrations of Ac-B-en was mixed with $2 \mathrm{ml}$ of TBA-TCA reagent $(15 \%$, w/v TCA; $0.375 \%, \mathrm{w} / \mathrm{v} \mathrm{TBA}$; and $0.25 \mathrm{M} \mathrm{HCl})$. The mixture was boiled in a water bath for $30 \mathrm{~min}$. After cooling, the suspension was centrifuged at $1500 \mathrm{~g}$ for $10 \mathrm{~min}$. The supernatant was then separated, and absorbance was measured at $535 \mathrm{~nm}$. The results were expressed as a simple concentration of MDA (nmol $/ 10^{8}$ sperm) as determined by the specific absorbance coefficient $\left(1.56 \times 10^{5} / \mathrm{mol}\right.$ per $\left.\mathrm{cm}^{3}\right)$.

MDA produced $(\mu \mathrm{mol} / \mathrm{ml})=\frac{\mathrm{OD} \times 10^{6} \times \text { total volume }(3 \mathrm{ml})}{1.56 \times 10^{5} \times \text { test volume }(1 \mathrm{ml})}$

$$
=\frac{\mathrm{OD} \times 30}{1.56}
$$

\section{Effect on L. acidophilus in vitro}

The effect of Ac-B-en on the growth of L. acidophilus was evaluated in vitro. Lyophilized L. acidophilus obtained from Lactobacil, Organon India Ltd, Gujarat, India, and was cultured in sterile Lactobacilli MRS broth medium maintained at $37{ }^{\circ} \mathrm{C}$ and $5 \% \mathrm{CO}_{2}$ for $24 \mathrm{~h}$ before each experiment. Briefly, culture tubes were prepared with $2 \mathrm{ml}$ of autoclaved MRS broth to which was added $100 \mu \mathrm{l}$ of vehicle as control or Ac-B-en solution to a final tube concentration of $120 \mu \mathrm{g} / \mathrm{ml}$ and $1.2 \mathrm{mg} / \mathrm{ml}$, which were representative of $1 \times \mathrm{MEC}$ and $10 \times$ MEC, respectively. N-9 was used at $550 \mu \mathrm{g} / \mathrm{ml}$ as a positive control. The tubes were inoculated with spores of $\sim 10^{-5}$ L. acidophilus population. The culture tubes were maintained at $37^{\circ} \mathrm{C}$ in an atmosphere containing $5 \% \mathrm{CO}_{2}$ and $95 \%$ air for a total period of $36 \mathrm{~h}$ with intermittent measurement of optical density (turbidity) at $600 \mathrm{~nm}$ at $6 \mathrm{~h}$ intervals. This experiment was performed five times in duplicates. Results are presented as mean \pm s.D.

\section{Mutagenicity test}

Mutagenicity of the test substance was assessed in a bacterial reverse mutation assay by using a preincubation method (Maron \& Ames 1983) that detects frameshift mutagens (Salmonella strains TA 97a and TA 98), bp mutagen (TA 100), or both (TA 102). The test was conducted following preincubation in the presence or absence of S9 mix without or with Ac-B-en at doses of 84,840 , and $8400 \mu \mathrm{g} /$ plate that represented final tube concentration of $1 \times, 10 \times$, and $100 \times$ of MEC. Ac-B-en was diluted in DMSO, which also served as the negative control. In experiments without external metabolic activation, 4-nitrophenylene diamine ( $20 \mu \mathrm{g} /$ plate) served as the positive control for TA 97a and TA 98, while sodium azide $\left(\mathrm{NaN}_{3} ; 1.5 \mu \mathrm{g} /\right.$ plate $)$ and daunomycin $(6 \mu \mathrm{g} /$ plate $)$ were used for TA100 and TA102 respectively. For experiments with metabolizing enzyme system, however, benzo( $\alpha)$ pyrene ( $1 \mu \mathrm{g} /$ plate) served the purpose for all strains. For each treatment, $0.1 \mathrm{ml}$ of the test substance, negative control, or positive control solution were mixed with $0.5 \mathrm{ml}$ of $0.1 \mathrm{~mol} / \mathrm{l}$ sodium PB ( $\mathrm{pH} 7.4$ ) in a sterilized tube, to which $0.1 \mathrm{ml}$ of the respective bacterial suspension $\left(\sim 10^{8}\right.$ bacteria/ml $)$ was subsequently added. Assays with metabolic activation involved substitution of $0.1 \mathrm{~mol} / \mathrm{l}$ sodium $\mathrm{PB}$ with $0.5 \mathrm{ml}$ of $\mathrm{S} 9 \mathrm{mix}$. The mixture was incubated with gentle shaking for $20 \mathrm{~min}$ at $37^{\circ} \mathrm{C}$ (pre-incubation). After this incubation period, $2 \mathrm{ml}$ top agar $(0.5 \%$ agar dissolved in $0.5 \%$ sodium chloride in doubledistilled water supplemented with $0.05 \mathrm{mM}$ D-biotin/L-histidine solution) was added and transferred to a petridish $(85 \mathrm{~mm}$ in diameter) with 'minimal agar' (1.5\% agar with 5\% glucose and $4 \%$ Vogel-Bonner Medium-E in double-distilled water). After the overlaid agar had solidified, the plates were incubated for $48 \mathrm{~h}$ at $37^{\circ} \mathrm{C}$ and the numbers of revertant colonies were subsequently evaluated manually. The level of microbial toxicity was confirmed for background lawn with a stereoscopic microscope. All samples were tested five times in two independent experiments. Results are presented as mean number of revertants \pm s.D., and the statistical analysis was done by one-way ANOVA.

\section{Statistical analysis}

The results were analyzed by paired two tailed $t$-test, $\chi^{2}$-test, and one-way ANOVA, as applicable, using the GraphPad Prism 3.0 software (GraphPad Software, Inc., San Diego, CA, USA). $P<0.05$ was considered significantly different.

\section{Declaration of interest}

The authors declare that there is no conflict of interest that could be perceived as prejudicing the impartiality of the research reported.

\section{Funding}

This research was a part of the network project of Council of Scientific of Industrial Research, New Delhi, India (COR 0010), and involved no additional financial support.

\section{Acknowledgements}

We are grateful to Dr A K Giri, Scientist of Molecular Human Genetics Department, Indian Institute of Chemical Biology, Kolkata, India, for providing the Salmonella strains. HOS test kit was a generous gift from Dr M M Misro and Dr S P Chaki from NIHFW, New Delhi. We would like to thank Mr S N Dey for his help in the electron microscopic studies.

\section{References}

Buege JA \& Aust SD 1978 Microsomal lipid peroxidation. In Methods in Enzymology, vol 52, pp 302-10. Eds S Fleischer \& L Packer. London: Academic Press.

Campbell SM, Crowe SM \& Mak J 2001 Lipid rafts and HIV-1: from viral entry to assembly of progeny virions. Journal of Clinical Virology 22 217-227. 
Chaudhary A, Mahajan K \& Singh RV 2007 Antifertility and antimicrobial studies of pharmaceutically important organolead (IV) complexes of phenanthrolines. Applied Organometallic Chemistry 21 117-127.

Clara A, Manjramkar D \& Reddy V 2004 Preclinical evaluation of magainin-A as a contraceptive antimicrobial agent. Fertility and Sterility 81 1357-1365.

Flajshans M, Cosson J, Rodina M \& Linhart O 2004 The application of image cytometry to viability assessment in dual fluorescence-stained fish spermatozoa. International Journal of Cell Biology 28 955-959.

Farnsworth NR \& Waller DP 1982 Current status of plant products reported to inhibit sperm. Research Frontiers in Fertility Regulation 2 1-16.

Garner DL \& Johnson LA 1995 Viability assessment of mammalian sperm using SYBR-14 and propidium iodide. Biology of Reproduction 53 276-284.

Halder B, Pramanick S, Mukhopadhyay S \& Giri AK 2005 Inhibition of benzo[a]pyrene induced mutagenicity and genotoxicity by black tea polyphenols theaflavins and thearubigins in multiple test systems. Food and Chemical Toxicology 43 591-597.

Hawes SE, Hillier SL, Benedetti J, Stevens CE, Koutsky LA, Hanssen P \& Holmes K 1996 Hydrogen peroxide producing lactobacilli and acquisition of vaginal infections. Journal of Infectious Diseases $\mathbf{1 7 4}$ 1058-1063.

Jayendran RS, Van der Ven HH, Perag-Pelaez M, Crab BG \& Zaneveld LJD 1984 Development of an assay to assess the functional integrity of the human sperm membrane and its relationship to other semen characteristics. Journal of Reproduction and Fertility 70 219-228.

Kabir SN, Ray HN, Pal BC \& Mitra D 2008 Pharmaceutical composition having virucidal and spermicidal activity. USPTO Application \#: 20080300197.

Kempf C, Jentsch P, Barre-Sinoussi FB, Poirier B, Morgenthaler JJ, Morell A \& Germann D 1991 Inactivation of human immunodeficiency virus (HIV) by low $\mathrm{pH}$ and pepsin. Journal of Acquired Immune Deficiency Syndromes 4 828-830.

Klebanoff SJ \& Coombs RW 1991 Viricidal effect of Lactobacillus acidophilus on human immunodeficiency virus type-1: possible role in heterosexual transmission. Journal of Experimental Medicine $\mathbf{1 7 4}$ 289-292.

Lambert PC, Peters C \& Centurion SA 2004 Mutagenicity of vaginal spermicides containing nonoxynol-9 in a bacterial assay. Journal of Reproductive Medicine 49 817-824.

de Lamirande E, Leclerc P \& Gagnon C 1997 Capacitation as a regulatory event that primes spermatozoa for the acrosome reaction and fertilization. Molecular Human Reproduction 3 175-194.

Maron DM \& Ames BN 1983 Revised methods for the Salmonella mutagenicity test. Mutation Research 113 173-215.

Nandi B, Roy S, Bhattacharya S \& Sinha Babu SP 2004 Free radicals mediated membrane damage by the saponins Acaciaside-A and Acaciaside-B. Phytotherapy Research 18 191-194.
Pakrashi A, Ray H, Pal BC \& Mahato SB 1991 Sperm immobilizing effect of triterpene saponins from Acacia auriculiformis. Contraception 43 475-483.

Pal DC 1996 Traditional systems of medicine in India. In Medicinal Plants, Herbal Drugs and Rural Health, pp 48-51. Eds SC Pakrashi, RN Mukherjee \& S Mukhopadhyay. Calcutta: Max Mueller Bhavan.

Richardson BA, Voeller B \& Anderson DJ 1998 Use of nonoxynol-9 and changes in vaginal lactobacilli. Journal of Infectious Diseases $\mathbf{1 7 8}$ $441-445$.

Sander FV \& Cramer SD 1941 A practical method for testing the spermicidal action of chemical contraceptives. Human Fertility 6 134-153.

Setty BS, Kamboj VP, Garg HS \& Khanna NM 1976 Spermicidal potential of saponins isolated from Indian medicinal plants. Contraception 10 135-143.

Sinha Babu SP, Sarkar D, Ghosh NK, Saha A, Sukul NC \& Bhattacharya S 1997 Enhancement of membrane damage by saponins isolated from Acacia auriculiformis. Japanese Journal of Pharmacology 75 $451-454$.

Smits AK, Goergen CA \& Delaney JA 1999 Contraceptive use and pregnancy decision making among women with HIV. AIDS Patient Care and STDS 13 739-746.

Souada K, Alib S, Mounirb A \& Mounira TM 2007 Spermicidal activity of extract from Cestrum parqui. Contraception 75 152-156.

Suleiman SA, Ali ME, Zaki MS, Malik EMEA \& Nast MA 1996 Lipid peroxidation and human sperm motility: protective role of vitamin $\mathrm{E}$. Journal of Andrology 17 530-537.

Vásquez A, Jakobsson T, Ahrné S, Forsum U \& Molin G 2002 Vaginal Lactobacillus flora of healthy Swedish women. Journal of Clinical Microbiology 40 2746-2749.

Wilborn WH, Hahn DW \& McGuire JJ 1983 Scanning electron microscopy of human spermatozoa after incubation with the spermicide nonoxynol-9. Fertility and Sterility 39 717-719.

Windebank S, Malcomber S, Maskell S, Scott A \& Carmichael P 2006 Validation of a high throughput bacterial screen for mutagenicity: incorporation of Salmonella typhimurium strain TA102. Toxicology 226 72-73.

World Health Organization (WHO) 1999 Laboratory Manual for the Examination of Human Semen and Cervical Mucus Interaction, edn 4. New York: Cambridge University Press.

Received 3 February 2009

First decision 10 March 2009

Revised manuscript received 21 May 2009

Accepted 19 June 2009 